\title{
System Design and Analysis for Maximum Consuming Power Control in Smart House
}

\author{
Saher Umer, Mineo Kaneko, Yasuo Tan, and Azman Osman Lim \\ Japan Advanced Institute of Science and Technology (JAIST), Ishikawa, Japan \\ Email: \{saher, mkaneko, ytan, aolim\}@jaist.ac.jp
}

\begin{abstract}
The efficiency, reliability, and stability of the smart house is expected to be significantly improved via home energy management system (HEMS), and smart meter (SM) is the most essential part of the energy system used to connect individual home appliance to the energy management system. As more and more home appliances and consumer electronics are deployed, the power consumption in smart house (1) tends to grow and (2) leads an increase in the risk of power blackout. In this paper, we propose a system design for controlling maximum consuming power and preventing home blackout. Proposed system consists of SM and home appliances, and we assume that SM will provide access to the power generated and consumed of the appliance, but with a time delay. Then, we analyze the resulting design to illustrate the impact of SM, for two important properties of the smart house, preventing power blackout and maintaining power system stability. We also present the simulation results which show the appliance power consumption behavior when the available power is limited. Simulation results also help us in applying stability test with stable/unstable and overshoot/non-overshoot regions.
\end{abstract}

Index Terms - home energy management system, smart meter, power supply, power demand, power system stability

\section{INTRODUCTION}

Smart home is an integration of home automation equipment, home securities, home communication and other attractive features based on advanced technologies, such as combined automation, combined networks and communication technologies [1], [2]. The objective of moving towards to the smart home is enhancing safety, energy efficiency, comfortable, economical and environment-friendly unique life style for each individual. In this way, we have to face the worst situation of energy shortage due to increasing number of consumer electronics and their attractive functions according to the development of smart home [3].These increased power consumption resources have been forcing the human to search methods for managing power to reduce power consumption in home because power used in the home is a typical example of consumptive one.

The high power required by home appliances (like white goods, audio/video devices and communication equipment and air conditioning systems), makes our

Manuscript received June 1, 2013; revised August 6, 2013. homes one of the most critical areas for the impact of power consumption. As the residential areas are one of the major power consumers today, reducing power in house would contribute greatly to the environment. As more and more home appliances and consumer electronics are deployed, the power consumption in home area (1) tends to grow and (2) leads an increase in the risk of power blackout [4]. As a result, we need energy management system in home which is responsible for monitoring and managing the working operation of home appliances [5].

The technology to manage and balance the home energy use is well-known as a home energy management system (HEMS) [6]. The HEMS is a networked system which is responsible for monitoring and managing the working operation of in-home appliances, and helps residences in order to reduce power consumption according to the specific requirements [7].The introduction and availability of SM technologies have introduced a paradigm shift in the analysis of power system [8].The real time monitoring and measuring of power consumption levels of home appliances through $\mathrm{SM}$ is a key and an integral part of smart house.

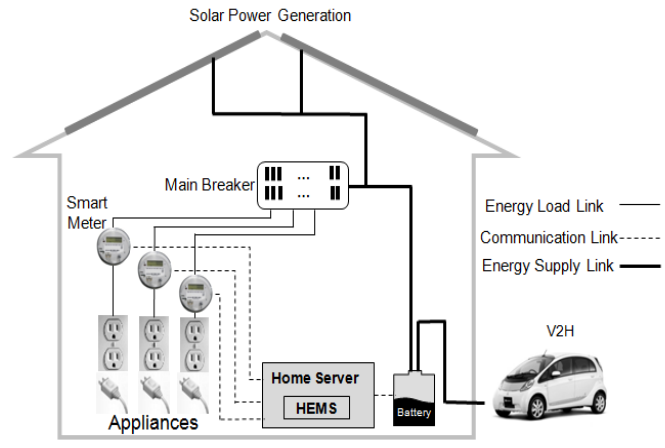

Power supply to residential load

Figure 1. A Sustainable energy house model with source and loads.

In this paper, we focus on the blackout prevention for the smart house and implementation of stability test. The detailed objectives of this study are to propose a system design consisting of SM and home appliances and then analyze the proposed system for power blackout prevention by introducing the system parameters. In overall scenario, the use of SM will help us in measuring the real-time power levels of appliances and send this information to HEMS then based on this information HEMS will provide the remaining available power 
information to the appliances, but with a time delay. In this paper, we also present the simulation results for the better understanding of how proposed system design work and what is the relationship between system parameters with each other. In last, we apply stability test to our proposed system.

The rest of this paper is organized as follows. Research background and motivation that are related to this paper summarized in Section 2. In Section 3 and 4, we described the system modeling and simulation results respectively. Stability analysis is presented in Section 5. Finally, we conclude our research and future works in Section 6.

\section{RESEARCH BACKGROUND AND MOTIVATION}

Modern society is greatly dependent on electricity due to development of information and urbanization. Electricity as an easy to use power is one of the most widely used energy all over the world. Electric power system is the basic need for daily life and its failure will probably paralyze the individual smart house to the whole city depending on the scale of a blackout. The severe accidents by a large scale blackout have been well recognized and many efforts have been done for preventing such a large scale blackout [9]-[11].On the other hand, the blackout in a smart house is also a critical problem, but no research study has been done for blackout prevention for home.It causes mental stress for the home user, and makes the smart house unstable. The future smart house should be stable for all the time, so we need to introduce blackout prevention for the smart house. In order to prevent a blackout, we need to control the instantaneous peak power.As each appliance behave on different time constant and it is hard to control fully the individual power consumption of home appliance by HEMS alone. So, we introduce additional functionality to each appliance for controlling the total energy consumption at home.

In our system design, there are two key parameters (1) information delay and (2) divisor of available power and these parameters effect the critical system behaviors such as stability and overshoot of energy waveform. Therefore, we need to carefully design these parameters so that system would be stable and has no overshoot, which will be discussed in section 4 and 5 .

\section{SYSTEM MODELLING}

Main breaker of the house is the breaker that the feeder wire connects to. Main breaker is designed to trip if the amperage rating exceeds the value labeled on the breaker. Main breaker is further divided into sub-breakers. Subbreakers are used to extend a feed for multiple branch circuits in whole area of house. From there, the sub breaker connects to outlets, lighting and other loads via individual circuit breakers. In Fig. 2, the system overview which we consider has one SM connected with mhome appliances. The use of SM will help us in measuring the actual real-time power consumption levels of appliances called $D_{i}(t)$.
Main Breaker

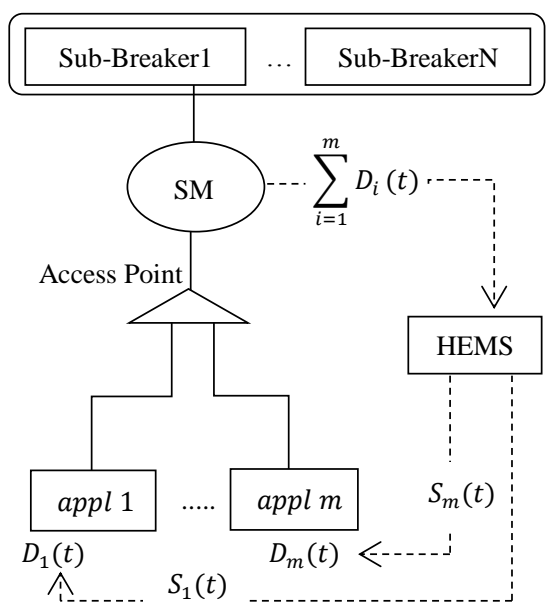

Figure 2. System overview considered in this paper.

In our proposed model, $\mathrm{SM}$ is responsible for collecting information about major consumed power level from all appliances and sends this information to HEMS. Then the functions of HEMS are to compute remaining available power for appliances, divide the remaining available power by $l$, and send this information i.e., $S_{i}(t)$ to appliances.lis the designer's parameter, also called divisor of available power. We assumed that the transfer of information of major power consumed level of $m$ appliances from SM to HEMS and then available remaining power level from HEMS to home appliance with some delay $\tau$. In our proposed modeling scheme, the total power available for all appliances is denoted as $P$, thus remaining power $R$ can be represented as:

$$
R(t)=P-\sum_{i=1}^{m} D_{i}(t)
$$

where $D_{i}(t)$ is the actual consumed power level of appliance $i$ at time $t$. On the other hand, we assume that each appliance has its own best performance power level $D_{\text {imax }}$, and it behaves based on the first order state equation given by

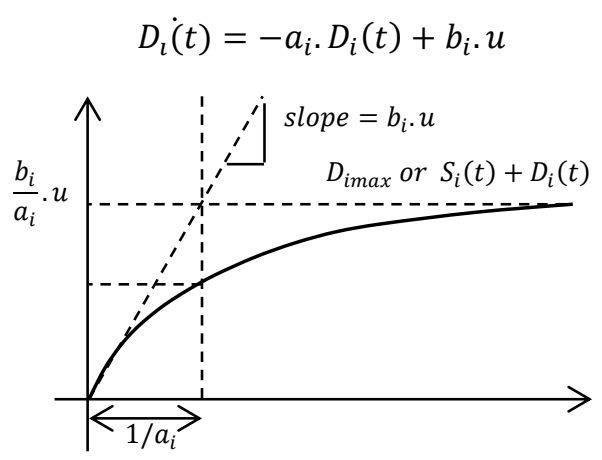

Figure 3. Simplified first-order power consumption profile of home appliance.

where,

$$
b_{i} u= \begin{cases}a_{i} \cdot D_{\text {imax }} & \text { if } S_{i}(t)+D_{i}(t)>D_{\text {imax }} \\ a_{i} \cdot\left(S_{i}(t)+D_{i}(t)\right) & \text { if } S_{i}(t)+D_{i}(t)<D_{\text {imax }}\end{cases}
$$


With

$$
S_{i}(t)=\frac{R(t-\tau)}{l}
$$

Note that, in our first order state equation model, $\frac{b_{i} \cdot u}{a_{i}}$ is the final steady state value of the appliance $i$, and $\frac{1}{a_{i}}$ is the time constant, i.e., the time required for the power to reach $63 \%$ of the final steady state value (see Fig. 3). In our model, $S_{i}(t)+D_{i}(t)$ is considered as an available power assigned to each appliance, where $S_{i}(t)$ is thedelayed version of the remaining power divided by a designer's parameter $l$. If this available power is larger than $D_{\text {imax }}$, the appliance manages its own power toward its maximum performance power level. We call it "normal mode" operation. On the other hand, if the available power for the appliance is less than $D_{\text {imax }}$, then the appliance reduces its performance to $S_{i}(t)+$ $D_{i}(t)$.The latter case is called "save mode" operation.

\section{Simulation Results}

Stability is one of the most important issues in a system design. In our system, each appliance has the parameter $a_{i}$ which decides the response speed, the remaining power $R(t)$ is divided by a designer's parameter $l$, and the information on the remaining power is fed back to each appliance with time delay $\tau$. As the first experiment, we will investigate the parameter dependency for the system stability.

For simulation scenario of our proposed system, we consider three appliances attached with SM. The best performance power level of three appliances is $D_{1 \max }=$ $10 \mathrm{~A}, D_{2 \max }=7 \mathrm{~A}$, and $D_{3 \max }=5 \mathrm{~A}$. The total available power for appliances is $20 A$. At first, we consider the case when all three appliances are turned $\mathrm{ON}$ at the same time with same system parameter $a_{i}=10(i=1,2,3)$.

From Fig. 4 and 5, we observed that the total power characteristics of the proposed system have three convergence behaviors; stable, unstable, and overshoot. We conclude that as the value of system parameter $l$ increases system becomes more stable. We also try to find out the border value of $l$ to get stable/unstable and overshoot/non-overshoot regions and the results are given in Table. I.

TABLE I. Border estimation for stable/unstable, overshoot/non-overshoot region.

\begin{tabular}{|c|c|c|c|}
\hline$\tau$ & $a_{i}$ & Stable/Unstable & $\begin{array}{c}\text { Overshoot/ } \\
\text { Non-overshoot }\end{array}$ \\
\hline 0.2 & 3 & $l=0.72$ & $l=1.22$ \\
& 10 & $l=1.24$ & $l=1.90$ \\
& 30 & $l=1.49$ & $l=2.30$ \\
\hline 0.4 & 3 & $l=1.12$ & $l=2.00$ \\
& 10 & $l=1.45$ & $l=2.20$ \\
0.8 & 30 & $l=1.56$ & $l=2.35$ \\
\hline & 3 & $l=1.48$ & $l=2.23$ \\
& 30 & $l=1.60$ & $l=2.29$ \\
& 301.72 & $l=2.31$ \\
\hline
\end{tabular}

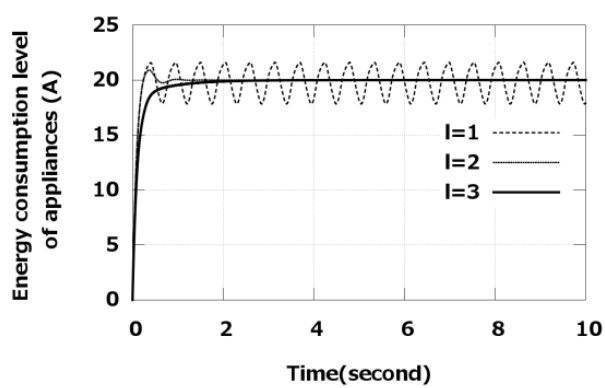

Figure 4. Total power characteristics for $l=1$ (unstable), $l=2$ (overshoot), and $l=3$ (stable) with $\tau=0.2$.

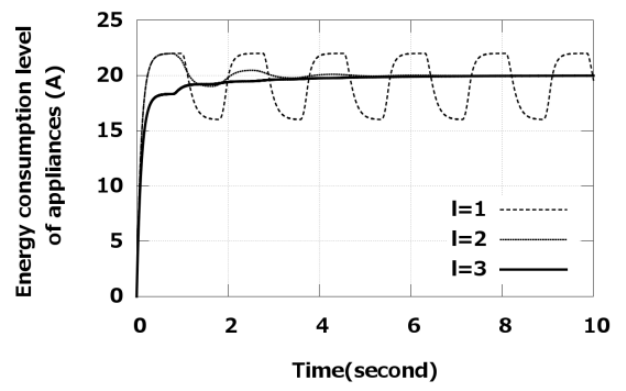

Figure 5. Total power characteristics for $l=1$ (unstable), $l=2$ (overshoot), and $l=3$ (stable) with $\tau=0.8$.

Above table gives, the border values of stable and overshoot are $l=0.72$ and $l=1.22$, respectively for which the system get stable within10 seconds. For the first case with $\tau=0.2$, when we use a larger value than 1.22 the system is stable and has no overshoot. If $l$ is in between 0.72 and 1.22 , the system is stable but has overshoot in total power consumption characteristics, and the system becomes unstable when $l$ is smaller than 0.72 .

As we can see from this table, the border in $l$ for stable/unstable and overshoot/non-overshoot increases as $a_{i}$ increases and as $\tau$ increases. So we need to decide the parameter $l$ by carefully estimating the maximum feedback time delay $\tau$ and the maximum speed of appliances.

As the second experiment, we will test that how the remaining power is distributed to appliances. In these simulations, we set $D_{1 \max }=D_{2 \max }=D_{3 \max }=$ $10 \mathrm{~A}, l=5$ and the total available poweris $20 \mathrm{~A}$.First, we have tested the effect of parameter $a_{i}$ to the final available power sharing among appliances. We consider the system parameter $a_{i}$ for all three appliances with same value and different value as well. The behaviors of the system with these criteria are as follows:

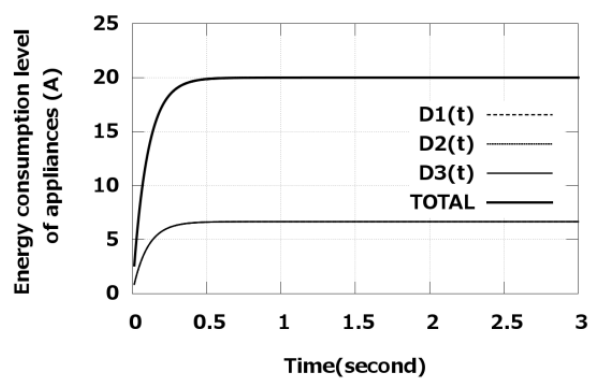

Figure 6. Power consumption behavior of appliances for $a_{i}=$ 10 (same turn ON time). 


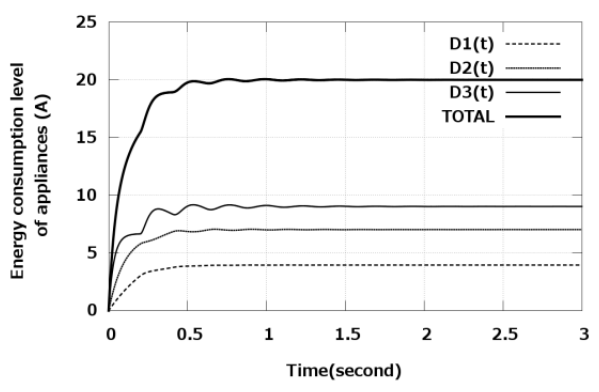

Figure 7. Power consumption behavior of appliances for $a_{1}=3$, $a_{2}=10$, and $a_{3}=30$ (same turn ON time).

As in Fig. 6, we can notice that all three appliances have same value for $a_{i}$ and $D_{\text {imax }}$. We observed that all three appliances shared the remaining power equally. However, in Fig. 7, we consider the situation when the value of time constant $a_{i}$ is different and $D_{\text {imax }}$ is kept same for all appliances. In this scenario, we observed that the appliance with small time constant takes more power from the available power level.

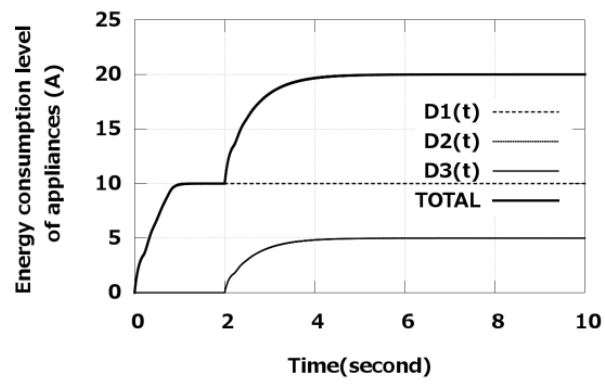

Figure 8. Power consumption behavior of appliances with different turn ON time ( $t=0$ for appliance1, $t=2$ for appliance 2 and 3 ).

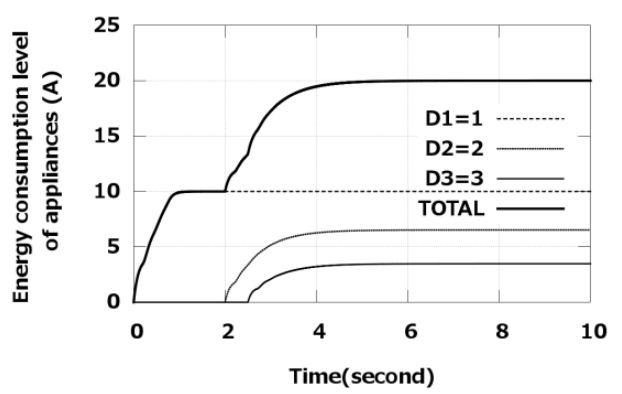

Figure 9. Power consumption behavior of appliances with different turn ON time ( $t=0$ for appliance $1, t=2$ for appliance 2 and $t=2.5$ for appliance3).

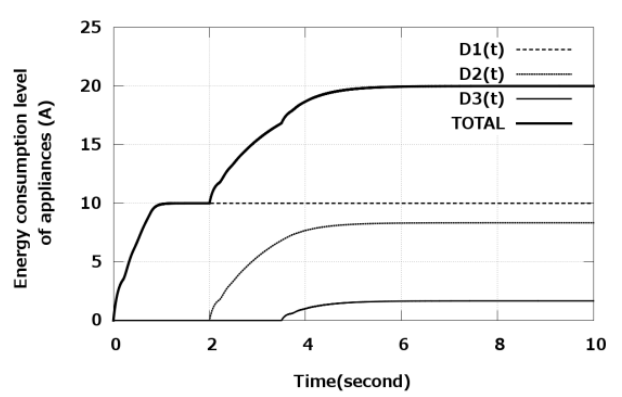

Figure 10. Individual appliance power consumption behavior with different turn ON time ( $t=0$ for appliance $1, t=2$ for appliance 2 and $t=3.5$ for appliance 3$)$.
Next we have tested the effect of turn ON timing to the final available power sharing. In Fig. 8, Fig. 9, and Fig. 10 , all three appliances have same $D_{\text {imax }}=10$ and $a_{i}=$ 10. The appliance which turned ON first can use more available power and the last turned $\mathrm{ON}$ appliance have smallest part of available power.

As we can see from two types of simulations, the final available power sharing among appliances is determined by the speed of each appliance and by the turn ON timing in our current system design. If we consider some priority in available power sharing, we need to modify our system, which is remained as a future problem.

\section{STABILITY ANALYSIS}

Basically the proposed system is non-linear but for the detailed analysis for stability, we will move to linear system because of the powerful tools available for the linear system. The non-linear system situation would be more complex and difficult to solve. The first step in analyzing a nonlinear system is usually to linearize it. We assigned mode to the situations as "normal mode" and "save mode" when the available power for appliances is more than the appliances demand or less than the appliances demand respectively. We carry out stability analysis and show that indeed the stability is dependent on delay $\tau$. Our focus is on the power consumption of the home appliances, which can be determined by Eq. (1)

First, we consider the case when all $m$ appliances operate in "normal mode". In this situation all three appliances have enough available power to operate till best performance, so that no appliance will disturb the working of other appliance. The system equation can be represented in matrix notation as:

$$
\left[\begin{array}{c}
D_{1}(t) \\
\vdots \\
D_{m}(t)
\end{array}\right]=\left[\begin{array}{ccc}
-a_{1} & & 0 \\
& \ddots & \\
0 & & -a_{m}
\end{array}\right]\left[\begin{array}{c}
D_{1}(t) \\
\vdots \\
D_{m}(t)
\end{array}\right]+\left[\begin{array}{c}
a_{1} D_{1 \max } \\
\vdots \\
a_{m} D_{\max }
\end{array}\right]
$$

In above case, the HEMS are stable all the times and no need to apply stability test because appliances can be operated independently. In second case, we consider that all $m$ appliances operate in the "save mode". In this situation, one appliance behavior will affect the other appliance behavior because available power for appliances is not enough for their best performance power level. The system equation can be represented in matrix notation as:

$$
\begin{aligned}
& {\left[\begin{array}{c}
D_{1}(t) \\
\vdots \\
D_{m}(t)
\end{array}\right]=\left[\begin{array}{ccc}
-a_{1}+a_{1} & & 0 \\
& \ddots & \\
0 & & -a_{m}+a_{m}
\end{array}\right]\left[\begin{array}{c}
D_{1}(t) \\
\vdots \\
D_{m}(t)
\end{array}\right]+} \\
& {\left[\begin{array}{ccc}
-a_{1} / l & \cdots & -a_{1} / l \\
\vdots & \ddots & \vdots \\
-a_{m} / l & \cdots & -a_{m} / l
\end{array}\right]\left[\begin{array}{c}
D_{1}(t-\tau) \\
\vdots \\
D_{m}(t-\tau)
\end{array}\right]+\left[\begin{array}{c}
P_{1} \\
\vdots \\
P_{m}
\end{array}\right]} \\
& P_{i}=a_{i} \cdot \frac{P}{l}
\end{aligned}
$$

Note that the coefficient matrix in the first term becomes a zero matrix. The above linear time delay system equation can be analyzed with Theorem 1:

$$
\dot{x}=A_{0} x(t)+A_{1} x(t-\tau)
$$


Theorem 1[11]: Power model described in eq. (2) is asymptotically stable if there exist real symmetric matrix $P>0$ such that:

$$
\left[\begin{array}{ccc}
\frac{1}{\tau}\left(P \hat{A}_{0}+\hat{A}_{0}^{T} P+2 P\right) & -P A_{1} A_{0} & -P A_{1}^{2} \\
-A_{0}^{T} A_{1}^{2} P & -P & 0 \\
-\left(A_{1}^{2}\right)^{T} P & 0 & -P
\end{array}\right]<0
$$

Proof: The Lyapunov function $V$ defined as $V=x^{T} P x$ where $P$ is positive definite is a Lyapunov function which is shown by using Eq. (3) and the existence of a symmetric matrix $R(\theta)$ that along with $P$ satisfies the conditions

$$
\begin{gathered}
P \hat{A}_{0}+\hat{A}_{0}^{T} P+\int_{-2 \tau}^{0} R(\theta) d \theta<0 \\
{\left[\begin{array}{cc}
p P-R(\theta) & P \hat{A}(\theta) \\
\hat{A}^{T}(\theta) P & -P
\end{array}\right]<0 \text { for } 0 \leq \theta \leq 2 \tau}
\end{gathered}
$$

With the help of theorem 1, the time-delay system in (2) is stable provided the time delay $\tau$ is such that the LMI in (3) is satisfied [12].

Third, we consider the case when $k$ appliances work in "normal mode" and $m-k$ appliances work in "save mode", where, $m$ is the total number of appliances. Then system equation in matrix notation is as follows.

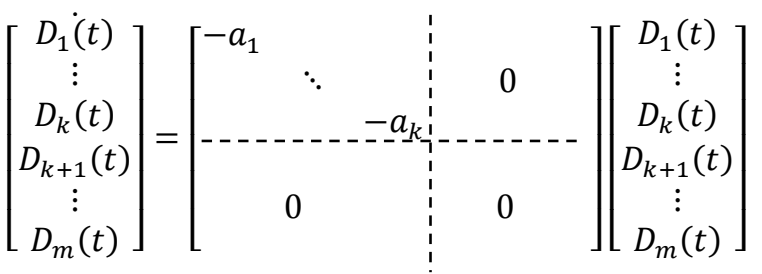

$$
+\left[\begin{array}{ccc}
0 & \ldots & 0 \\
\vdots & \ddots & \vdots \\
0 & \ldots & 0 \\
-\frac{a_{k+1}}{l} & \ldots & -\frac{a_{k+1}}{l} \\
\vdots & \ddots & \vdots \\
-\frac{a_{m}}{l} & \ldots & -\frac{a_{m}}{l}
\end{array}\right]\left[\begin{array}{c}
D_{1}(t-\tau) \\
\vdots \\
D_{k}(t-\tau) \\
D_{k+1}(t-\tau) \\
\vdots \\
D_{m}(t-\tau)
\end{array}\right]+\left[\begin{array}{c}
a_{1} D_{1 \max } \\
\vdots \\
a_{k} D_{k \max } \\
P_{k+1} \\
\vdots \\
P_{m}
\end{array}\right]
$$

Theorem 1 can be directly applied again to the third case to test stability.

\section{CONCLUSION AND FUTURE WORK}

The introduction of SM has brought a paradigm shift in the analysis of power consumption of home appliances in smart house. In this paper, we study the effect of SM that provides access to power supply and demand, but with some time delay. The main objectives of this paper are to prevent power blackout in home and maintain power stability of smart house by proposing a system design based on home appliances and SM. To reach above objectives, we analyzed the proposed system design in simulation environment. The simulation results showed the dependency of system parameters $a_{i}$ (appliance response speed), $D_{\text {imax }}$ (appliance maximum power consumption level), $l$ (divisor of available power) and $\tau$ (time delay in information transfer between SM to HEMS and HEMS to appliance) in system behavior such as stable/unstable, overshoot/non-overshoot.

From simulation analysis, we get the border estimation table representing the stable/unstable and overshoot/nonovershoot regions which help us in concluding that the border in $l$ for stable/unstable and overshoot/nonovershoot increases as $a_{i}$ increases and as $\tau$ increases. So, we need to decide the parameter $l$ by carefully estimating the maximum feedback time delay $\tau$ and the maximum speed of appliances. We also conduct couple of experiments that show how the remaining power is distributed to appliances and what is the effect of turn ON timing of the appliances to the final available power sharing. We noticed from two types of simulations, the final available power sharing among appliances is determined by the speed of each appliance and by the turn ON timing. For our future work, we will try to consider priority of appliances in available power sharing for this purpose we have to modify our system design.

\section{REFERENCES}

[1] H. Lin, J. Chen, M. jiang, and C. Huang, "Integration of GPRS and wireless LANs with multimedia applications," London: Springer Berlin Heidelberg, 2002, pp. 704-711.

[2] Y. Shi, W. Xie, G. Xu, E. Chen, and Y. Mao, "The smart classroom: Merging technologies for seamless tele-education," IEEE Pervasive Computing, vol. 2, pp. 47-55, January 2003.

[3] C. Reinisch, M. J. Kofler, and W. Kastner, "Think Home: A smart home as digital ecosystem," in Proc. IEEE International Conf. on Digital Ecosystems and Technologies, 2010, pp. 256-261.

[4] M. J. Kofler, C. Reinisch, and W. Kastner, "An intelligent knowledge representation of smart home energy parameters," EEE World Renewable Energy Congress, pp. 921-928, May 2011.

[5] J. Han, C. Choi, W. Park, and I. Lee, "Green home energy management system through comparison of energy usage between the same kinds of home appliances," in Proc. IEEE $15^{\text {th }}$ International Symposium on Consumer Electronics, 2011, pp. 1-4.

[6] Y. Son and K. Moon, "Home energy management system based on power line communication," in Proc. $28^{\text {th }}$ International Conf. on Consumer Electonics, 2010.

[7] M. Pipattanasomporn, M. Kuzlu, and S. Rahman, "An algorithm for intelligent home energy management and demand response analysis," IEEE Trans. on Smart Grid, 2012.

[8] A. Kiani and A. Annaswamy, "The effect of a smart meter on congestion and stability in a power market," in Proc. IEEE Conf. on Decision and Control, December 2010, pp.194-198.

[9] J. Barkans and D. Zalostiba, "New concept and solutions for prevention of power system blackout," in Proc. IET International Conf. on Development in Power System Protection, April 2010.

[10] J. Barkans and D. Zalostiba, "Blackout prevention and power system self-restoration," in Proc. IEEE International Conf. on Computer as Tool, September 2007, pp. 1547-1554.

[11] Z. Shaobo and S. Zhanhui, "Challenges and opportunities in emergency management of electric power system blackout," in Proc. International Conf. on E-Product E-Service and EEntertainment, 2010.

[12] K. Gu, V. Kharitonov, and J. Chen, "Stability of time delay system," in Control Engineering, Birkhuser Boston, June 2003.

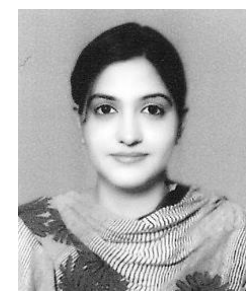

SaherUmerwas born in Lahore, Pakistan. She received her BCS (Bachelor of Computer Science) from AllamaIqbal Open University, Islamabad, Pakistan in 2004. She also received her MSc-IT (Masters in Information Technology) from University of the Punjab (PUCIT), Lahore, Pakistan in 2007. She is currently a Ph.D. student in Graduate School of Information Science, Japan Advanced Institute of Science 
and Technology (JAIST). Her areas of research include Home Energy Management System, Energy stability-aware scheme for HEMS, Renewable Energy contributions for HEMS and Smart metering technologies.

She also worked as a Visiting Lecturer at University of the Punjab and Education University, Lahore, Pakistan in 2010 and 2011 respectively.

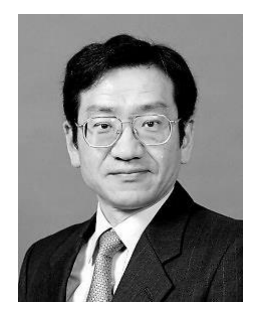

Mineo Kanekoreceived Bachelor of Engineering, Master of Engineering, and Doctor of Engineering degrees in Electrical and Electronic Engineering from Tokyo Institute of Technology in 1981, 1983, and 1986, respectively. From 1986 to 1996, he joined Tokyo Institute of Technology as a research associate, a lecturer, and an associate professor. In 1996, he transferred to Japan Advanced Institute of Science and Technology (JAIST), and currently he is a professor in the Graduate School of Information Science, JAIST. His research interests include circuit theory, computer aided design of VLSIs, and system optimization. He is a member of IEEE, ACM, IEICE, and IPSJ.

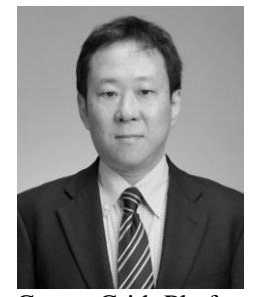

Yasou Tan received his Ph.D. from Tokyo Institute of Technology in 1993. Since then, he has been a member of the School of Information Science atJapan Advanced Institute of Science and Technology, and a professor since1997. He is interested in ubiquitous computing systems, especially homenetwork and smart home systems $\mathrm{He}$ is the chairman of New GenerationNetwork Promotion Forum Residential ICT SWG, and Green Grid Platform atHome alliance, and a fellow of ECHONET consortium. He is a member of IEEE,ACM, IPSJ, IEICE, IEEJ, JSSST and JNNS

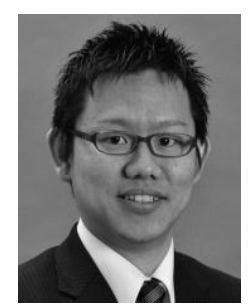

Azman Osman Lim received the B.Eng. (Hons) and M.Inf. Technology degrees from Universiti Malaysia Sarawak (UNIMAS), Malaysia in 1998 and 2000, respectively. He received the Ph.D. degree in communications and computer engineering from Kyoto University in 2005. He was a visiting researcher at Fudan University in China for two months. During 2005-2009, he was an expert researcher at National Institute of Information and Communications Technology (NICT), Japan. Since 2009, he has been working at Japan Advanced Institute of Science andTechnology (JAIST) as an associate professor. His research interests include multihop wireless networks, wireless sensor networks, home networks, wireless mesh networks, heterogeneous wireless networks, network coding, cyber-physical system. He is a member of IEEE, IEICE and IPSJ. 